\title{
Associations between essential medicines and health outcomes for cardiovascular disease
}

\author{
Liane Steiner $^{1}$, Shawn Fraser ${ }^{2}$, Darshanand Maraj ${ }^{1}$ and Nav Persaud ${ }^{1,3^{*}}$ (])
}

\begin{abstract}
Background: National essential medicines lists are used to guide medicine reimbursement and public sector medicine procurement for many countries therefore medicine listings may impact health outcomes.

Methods: Countries' national essential medicines lists were scored on whether they listed proven medicines for ischemic heart disease, cerebrovascular disease and hypertensive heart disease. In this cross sectional study linear regression was used to measure the association between countries' medicine coverage scores and healthcare access and quality scores.
\end{abstract}

Results: There was an association between healthcare access and quality scores and health expenditure for ischemic heart disease $(p \leq 0.001)$, cerebrovascular disease $(p \leq 0.001)$ and hypertensive heart disease $(p \leq 0.001)$. However, there was no association between medicine coverage scores and healthcare access and quality scores for ischemic heart disease $(p=0.252)$, cerebrovascular disease $(p=0.194)$ and hypertensive heart disease $(p=0.209)$ when country characteristics were accounted for.

Conclusions: Listing more medicines on national essential medicines lists may only be one factor in reducing mortality from cardiovascular disease and improving healthcare access and quality scores.

Keywords: Cardiovascular disease, Essential medicines, Amenable mortality

\section{Introduction}

Approximately $29 \%$ of deaths worldwide are from cardiovascular disease specifically, ischemic heart disease, stroke and hypertensive heart disease [1]. The burden of these and other non-communicable diseases (NCD) will be associated with productivity loss and catastrophic healthcare costs [2] which has the potential to significantly undermine national macroeconomic development [3]. Deaths from cardiovascular disease are amenable to healthcare including treatments such as antihypertensives [4].

Following a 2011 United Nations meeting, the World Health Organization (WHO) released a briefing

\footnotetext{
*Correspondence: nav.persaud@utoronto.ca

${ }^{3}$ Department of Family and Community Medicine, University of Toronto,

Toronto, ON, Canada

Full list of author information is available at the end of the article
}

document which stated that the burden of NCD's cannot be reduced without access to essential medicines [5]. Essential medicines are those that satisfy the priority health care needs of the population [6]. The purpose of an essential medicines list is to ensure quality medicines are available in a functioning health system, in appropriate forms, at affordable prices for both the individual and the community [6]. The WHO created a Model List of Essential Medicines (WHO Model List) which provides recommendations for minimum medicine needs for a basic health-care system. More than 100 countries have embraced the idea of essential medicines and adapted their own national essential medicines list (NEML) to address their health care priorities informed by their national burden of disease [2]. NEMLs are used to guide appropriate use of medicines, as well as medicine selection, reimbursement and public sector procurement $[7,8]$. In the public sector, essential medicines 
are more available than other medicines, suggesting that there may be preferential attention from governments given to them, therefore carefully selecting and adopting an NEML is the first step in ensuring equitable access to pharmaceutical treatment [2]. Medication availability and accessibility plays and important role in addressing the burden of NCD's [3] as evident by a reduction in mortality and morbidity in many countries since the implementation of essential medicines [9]. Population mortality that is amenable to care is assessed by the healthcare access and quality (HAQ) score that is available for 195 countries and that is comprised of 32 causes of death including ischemic heart disease, cerebrovascular disease and hypertensive heart disease [4].

The purpose of this study was to determine the relationship between listing essential medicines used to treat ischemic heart disease, cerebrovascular disease and hypertensive heart disease and amenable mortality related to these conditions measured by the HAQ score [4].

\section{Methods}

\section{Dataset sources}

All medications, with some exceptions, from countries' NEMLs hosted in the WHO's National Essential Medicines Lists Repository were extracted and recorded in an Excel database [10, 11]. NEMLs for 137 countries were identified [10].

We used the 2018 amenable mortality subscores, calculated by measuring age standardized mortality rates, for ischemic heart disease, cerebrovascular disease and hypertensive heart disease [4].

\section{Inclusion criteria}

Countries were included if they had a NEML captured by the Global Essential Medicines (GEM) database and a HAQ score for ischemic heart disease, cerebrovascular disease and hypertensive heart disease.

\section{Data collection}

In order to identify which medications were relevant to the three causes of interest (ischemic heart disease, cerebrovascular disease and hypertensive heart disease), we searched for guidelines for ischemic heart disease, cerebrovascular disease and hypertensive heart disease on the WHO website in June 2019. Four international guidelines distributed by the WHO, an internationally recognized health authority, were selected: Prevention and Control of Non-communicable Diseases: Guidelines for primary health care in low-resource settings [12], WHO Package of Essential Non-communicable Diseases Interventions for Primary Health Care in Low-Resource Settings [13], Technical Package for cardiovascular disease management in primary health care- evidence-based treatment protocols [7], Tackling NCDs: "Best Buys" and other recommended interventions for the prevention and control of non-communicable diseases [14]. Although it is not an internationally recognized guideline, additional guidance from the American Heart Association's website was used to ensure all relevant medicines were captured [15]. These guidelines along with the WHO Model List 20th edition [16] were used to identify medicines used for treatment of ischemic heart disease, cerebrovascular disease and hypertensive heart disease. Guidelines were searched using the causes and associated International Classification of Diseases 10th revision codes provided by the HAQ score [4].

Population size, health expenditure and life expectancy were retrieved from the Global Health Observatory [17]; prevalence for ischemic heart disease, cerebrovascular disease and hypertensive heart disease was retrieved from the Global Burden of Disease Study [1]. Most data was for the year 2016; if 2016 data was not available, data from the closest year to 2016 was retrieved. Country characteristics can be found in Table 1 .

\section{Data extraction}

Using the identified guidelines for ischemic heart disease, cerebrovascular disease and hypertensive heart disease, medications used to treat these conditions were abstracted. If a guideline indicated a therapeutic class of medicines, that class was fully expanded to include all medicines because medicines within the same chemical subgroup may be considered therapeutically similar. The WHO Model List recognizes interchangeability of certain medicines on their list for others within the same therapeutic class [16]. Using this principle, 4th level Anatomical Therapeutic Chemical Classification (ATC) codes [18] were used to guide which medicines are in the same therapeutic class. If a therapeutic class was mentioned and specific alternatives were stated, only those medicines were included (no therapeutic class expansion was done).

Medicines listed on the WHO Model List or those from guidelines appearing on the WHO Model List (in a form that is usable for the conditions or cause), with a square box symbol, were fully expanded based on the 4th level, chemical subgroup of the ATC code to include all medicines within that therapeutic class. If the medicine is not denoted with a square box it was not expanded. If specific medicines considered equivalent were stated, only those medicines were included. A medicine coverage score was created by summing the number of medicines 


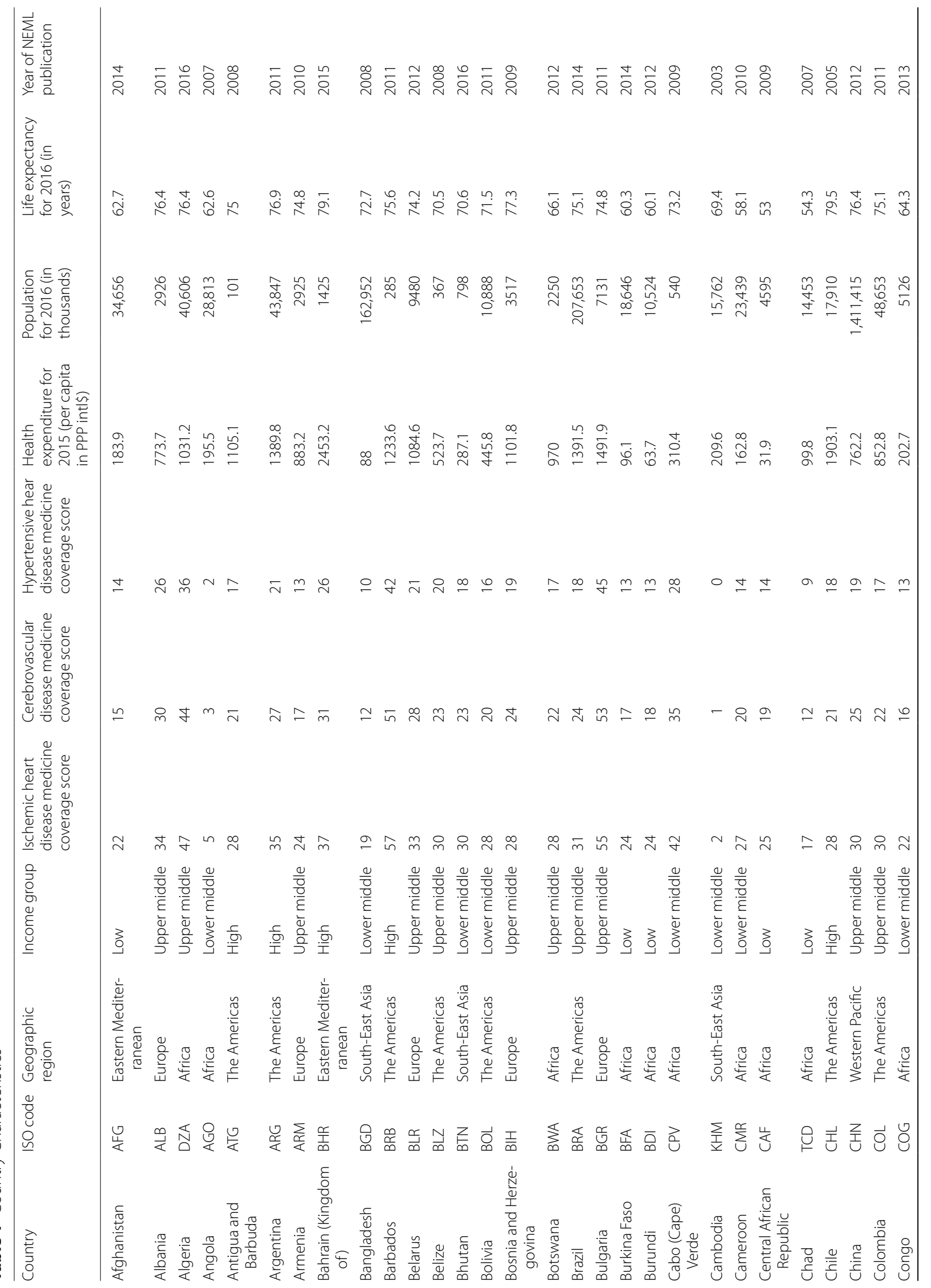




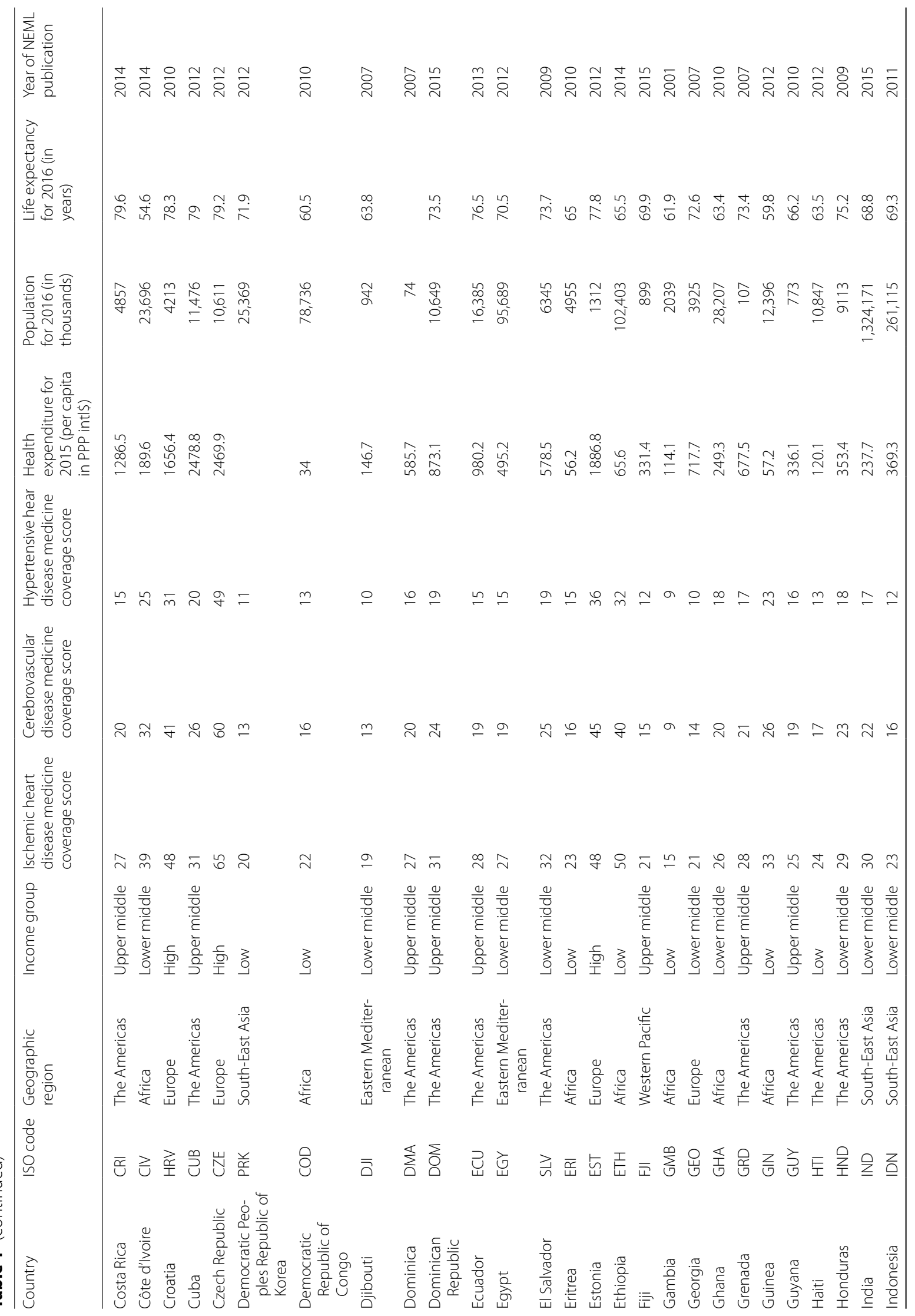




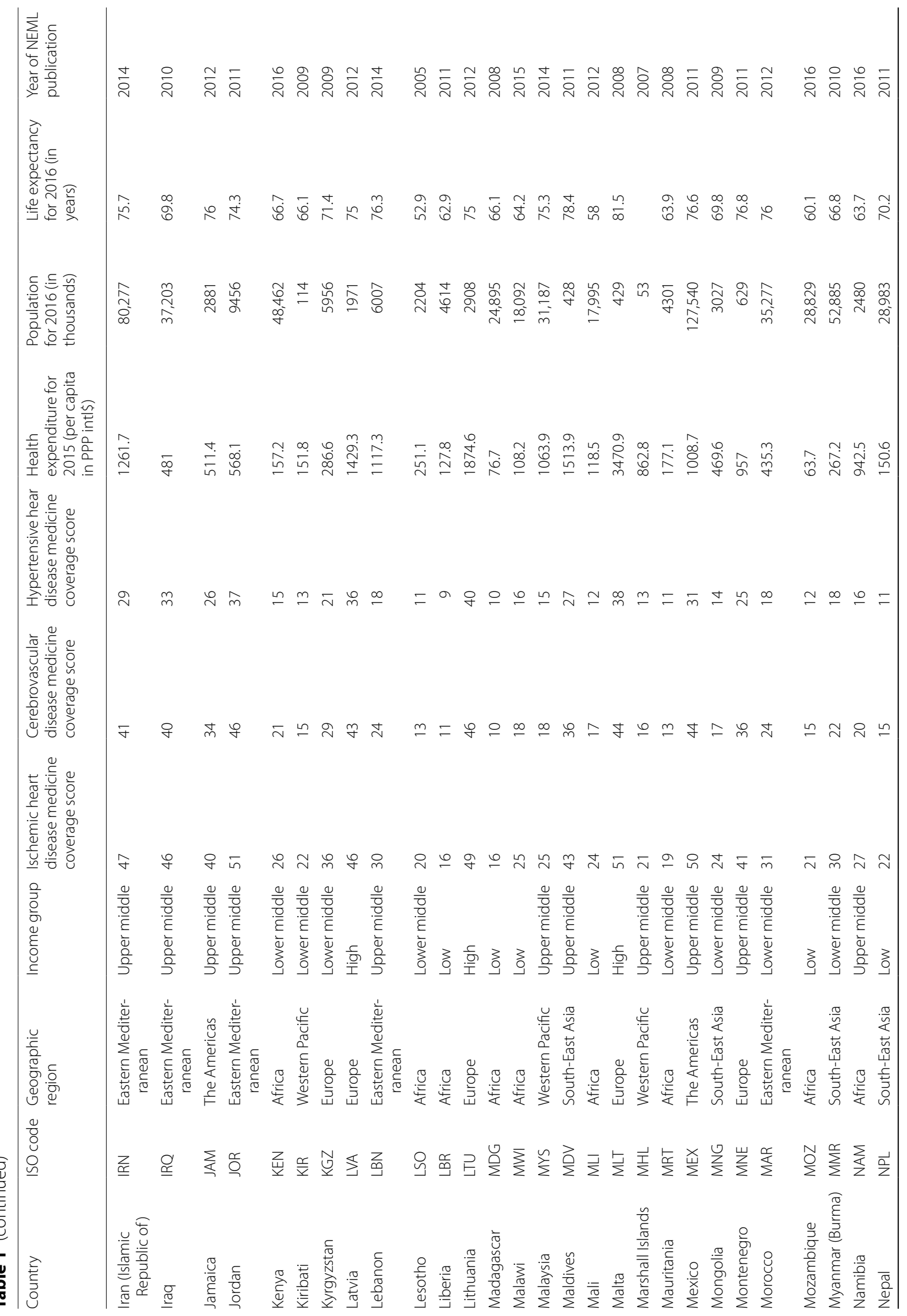




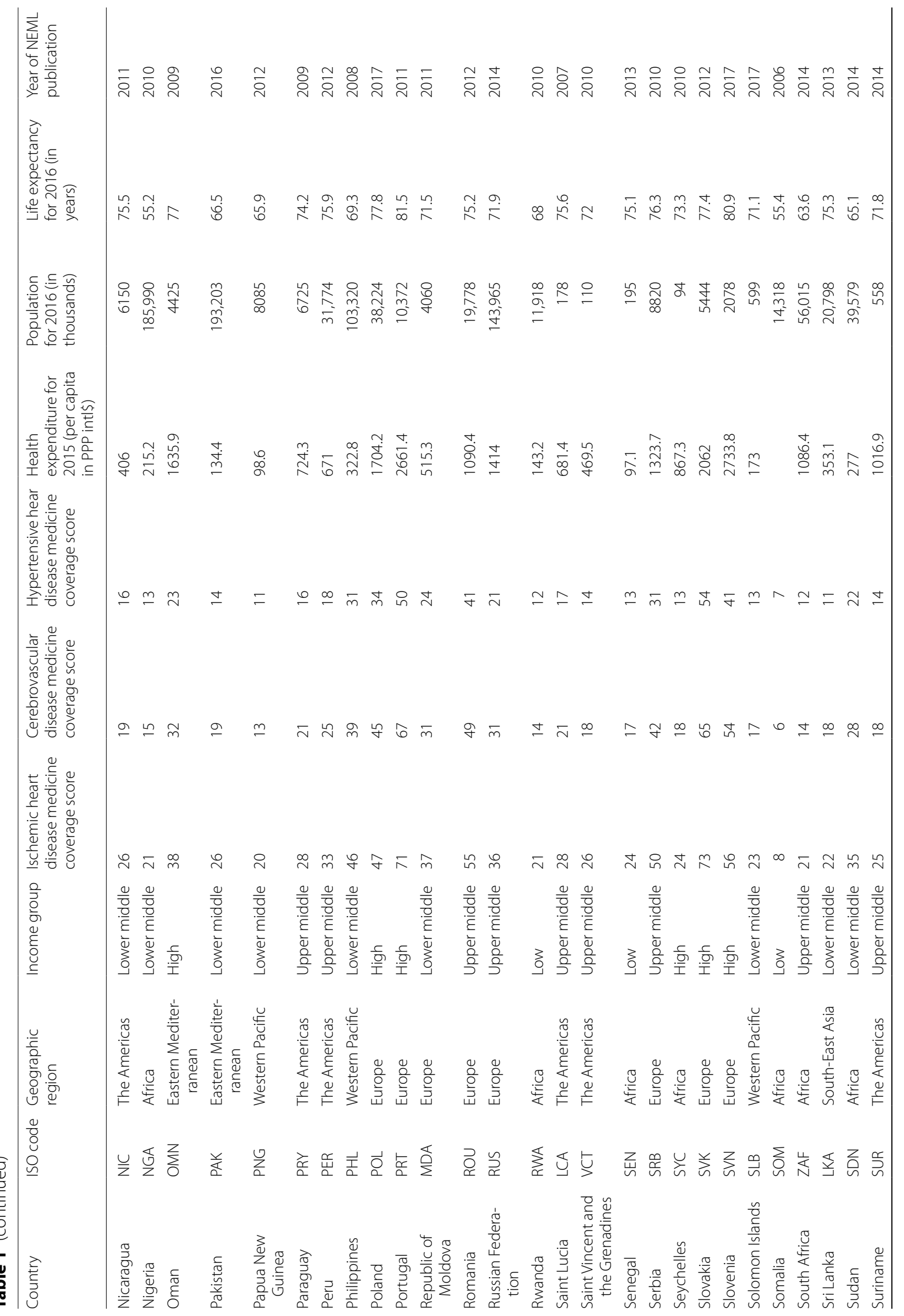




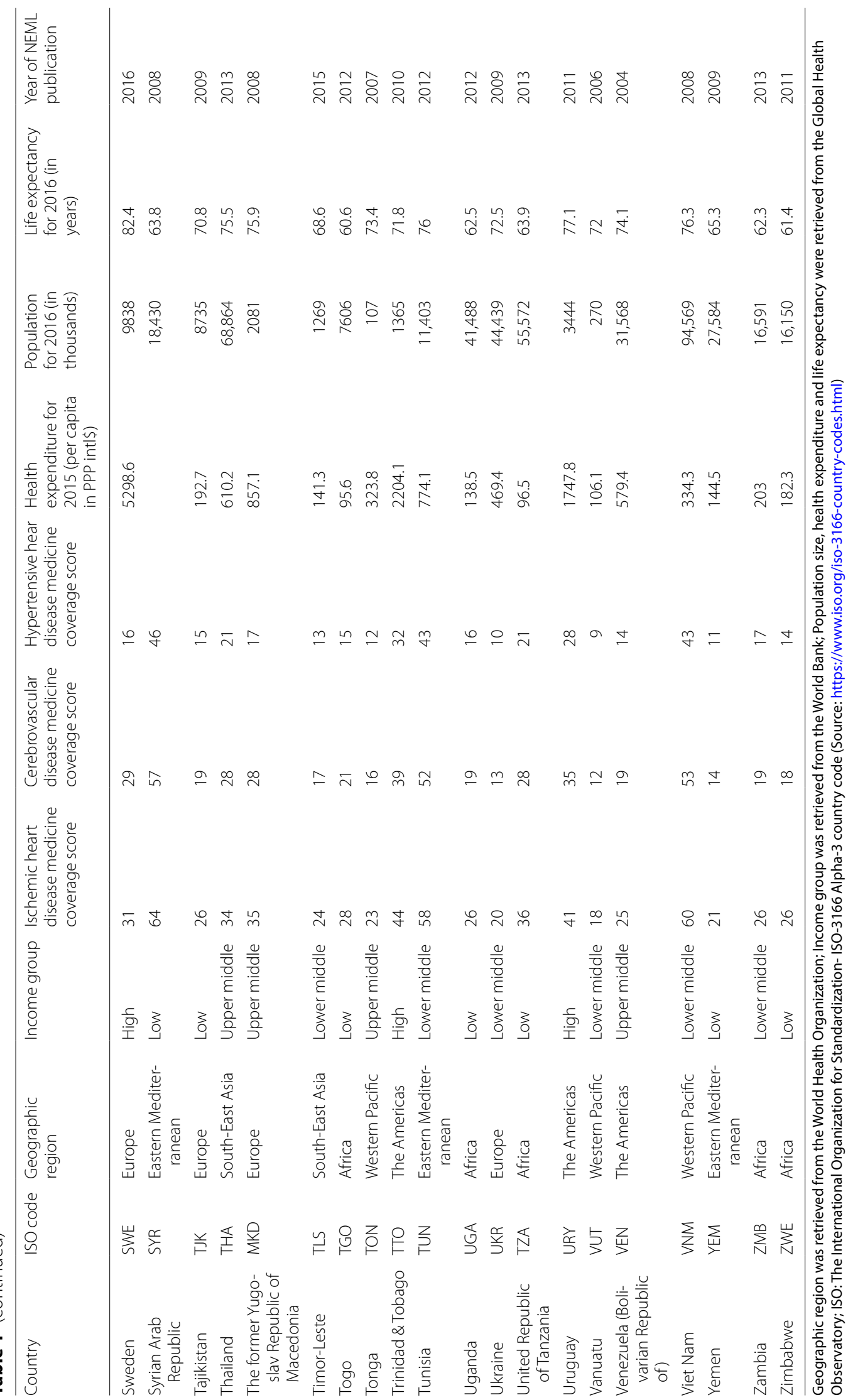


on a country's NEML that were also listed on our list of medicines used to treat each HAQ cause.

\section{Data analysis}

Data was analyzed using IBM SPSS Statistics version 26 (IBM Corp., 2018), and a p-value $\leq 0.05$ was considered significant. An ordinary least squares linear regression model was used to test the hypothesis that there would be a positive relationship between listing medicines (medicine coverage score) and HAQ scores. HAQ score was used as the dependent variable and the previously calculated medicine coverage score was used as the independent variable. Linear regression results are reported for both unadjusted and adjusted with health expenditure, population, life expectancy and prevalence as covariates.

\section{Results}

In total, 131 countries were included in the analysis having both a NEML and HAQ score (Table 1). WHO regions represented by countries were the Eastern Mediterranean (14 countries), Europe (26 countries), Africa
(38 countries), the Americas (29 countries), SouthEast Asia (13 countries) and the Western Pacific (11 countries) [17]. Using the World Bank categorization, included countries represented a range of income levels with 28 low income countries, 40 lower-middle income countries, 43 upper middle countries and 20 high income countries [19]. Three countries (Democratic Peoples Republic of Korea, Somalia and Syrian Arab Republic) were excluded from the regression analysis because they were missing values for healthcare expenditure.

The total number of medicines identified through guideline searches for each cause was 103 medicines for ischemic heart disease, 96 medicines for cerebrovascular disease and 73 medicines for hypertensive heart disease (see Additional file 1 for list of medicines). Figure 1 graphs the association between medicine coverage score and HAQ score, with health expenditure represented by bubble size.

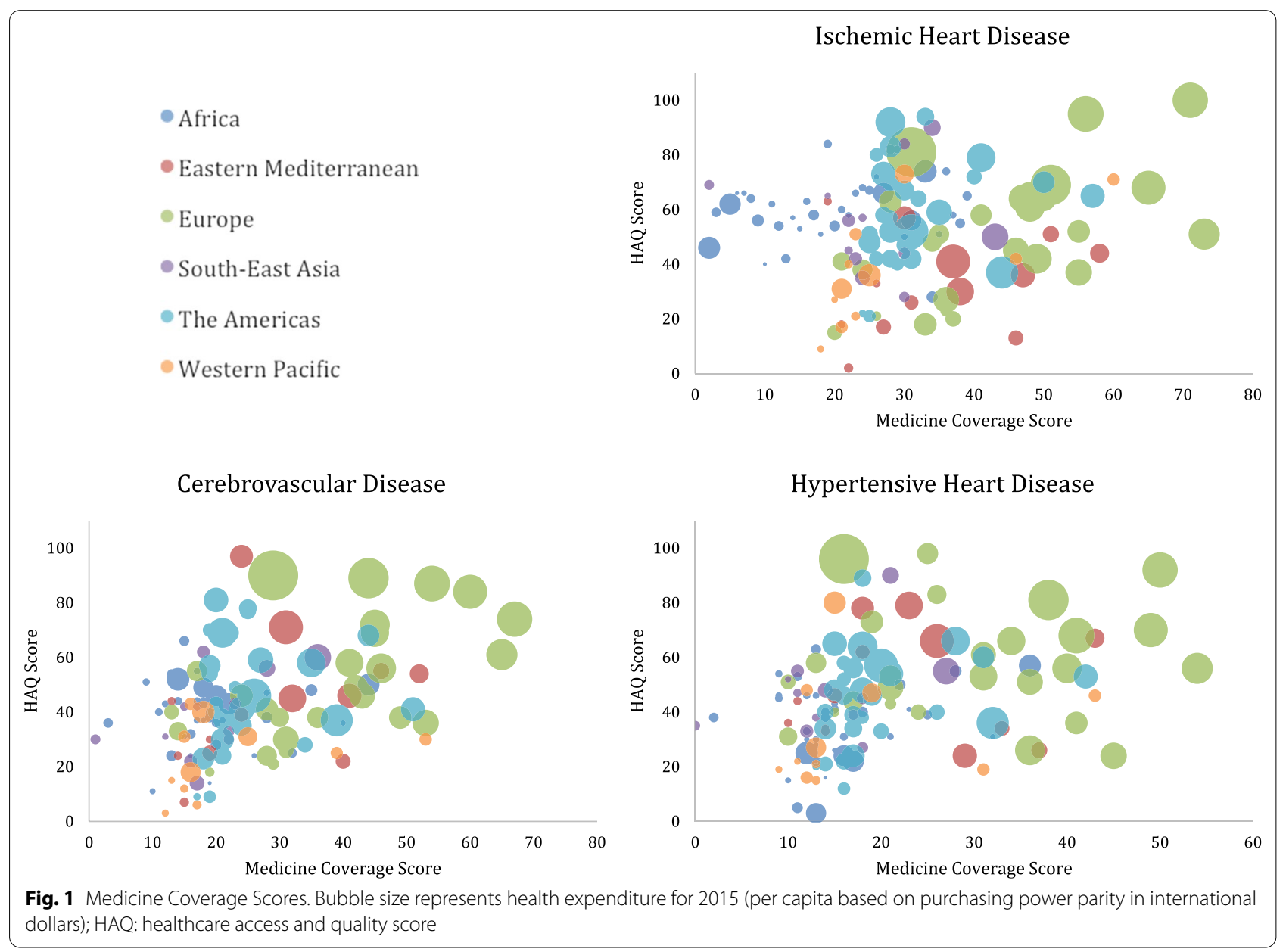




\section{Ischemic heart disease}

For ischemic heart disease, medicine coverage scores ranged from 2 to 73 (median: 28, IQR: 23 to 37). Results of the unadjusted linear regression model show that listing ischemic heart disease medicines only explained $0.5 \%$ of the variability in the HAQ scores across countries. After adjusting for population size, health expenditure, life expectancy and prevalence, approximately $18 \%$ of differences in the HAQ score for ischemic heart disease were explained. In the adjusted regression, there was no association between medicine coverage score and HAQ score for ischemic heart disease $(p=0.252)$, however other variables showed an association with HAQ score. Health expenditure was associated with a 0.011 point increase in HAQ score for each additional per capita dollar $(p<0.001)$ and prevalence of ischemic heart disease was associated with a 0.007 point decrease in HAQ score for each additional 100, 000 people diagnosed with ischemic heart disease $(p<0.001)$ (Table 2).

\section{Cerebrovascular disease}

For cerebrovascular disease, medicine coverage scores ranged from 1 to 67 (median: 21, IQR 17-31). Results of the unadjusted linear regression model show that listing cerebrovascular disease medicines explained approximately $15 \%$ of the variation in the HAQ scores. After adjusting for covariates approximately $44 \%$ of differences in the HAQ score for cerebrovascular disease were explained. In the unadjusted regression, there was an association between medicine coverage score and HAQ score for cerebrovascular disease $(p<0.001)$, however the relationship was not present when covariates were included $(p=0.194)$. Similar to ischemic heart disease, other variables in the adjusted analysis showed a significant association with HAQ scores. Health expenditure was associated with a 0.014 point increase in HAQ score for each additional per capita dollar $(p<0.001)$, life expectancy was associated with a 0.557 point increase with each additional year of life $(p=0.042)$ and prevalence of cerebrovascular disease was associated with a 0.008 point decrease in HAQ score for each additional 100, 000 people diagnosed with cerebrovascular disease $(p=0.001)$ (Table 3).

\section{Hypertensive heart disease}

For hypertensive heart disease, medicine coverage scores ranged from 0 to 54 (median 17, IQR 13-25). Results of the unadjusted linear regression model show that listing hypertensive heart disease medicines explained approximately $11 \%$ of the variation in the HAQ score. Results of the adjusted analysis show that approximately $45 \%$ of differences in HAQ score were explained. Similar to cerebrovascular disease, an association between medicine coverage score and the HAQ score was observed for hypertensive heart disease $(p<0.001)$, however the multivariate relationship was not present when covariates were

Table 2 Ischemic Heart Disease: Medicine Coverage Score

\begin{tabular}{|c|c|c|c|c|c|c|c|}
\hline & Variable & B & 95\% Cl lower bound & 95\% Cl upper bound & Beta & Pvalue & Pearson correlation \\
\hline Unadjusted & Medicine coverage score & 0.109 & -0.164 & 0.382 & 0.69 & 0.43 & 0.069 \\
\hline \multirow[t]{5}{*}{ Adjusted } & Medicine coverage score & 0.194 & -0.14 & 0.528 & 0.123 & 0.252 & 0.108 \\
\hline & Health expenditure & 0.011 & 0.005 & 0.017 & 0.467 & $<0.001$ & 0.232 \\
\hline & Population & $-1.056 \mathrm{E}-7$ & 0 & 0 & 0.001 & 0.991 & -0.008 \\
\hline & Life expectancy & 0.058 & -0.636 & 0.752 & 0.02 & 0.869 & 0.093 \\
\hline & Prevalence & -0.007 & -0.01 & -0.004 & -0.49 & $<0.001$ & -0.108 \\
\hline
\end{tabular}

$R_{\text {unadjusted }}^{2}=0.005(F=0.626,(d f 130), p=0.43) . R_{\text {adjusted }}^{2}=0.176(F=5.131$, (df 125), $p<0.001)$

$\mathrm{B}$, unstandardized coefficient; Beta, standardized coefficient; $\mathrm{Cl}$, confidence interval

Table 3 Cerebrovascular disease: medicine coverage score

\begin{tabular}{|c|c|c|c|c|c|c|c|}
\hline & Variable & $B$ & 95\% Cl lower bound & $95 \% \mathrm{Cl}$ upper bound & Beta & P-value & Pearson correlation \\
\hline Unadjusted & Medicine coverage score & 0.565 & 0.333 & 0.796 & 0.391 & $<0.001$ & 0.391 \\
\hline \multirow[t]{5}{*}{ Adjusted } & Medicine coverage score & 0.173 & -0.089 & 0.435 & 0.117 & 0.194 & 0.393 \\
\hline & Health expenditure & 0.014 & 0.009 & 0.018 & 0.587 & $<0.001$ & 0.609 \\
\hline & Population & $-3.977 \mathrm{E}-06$ & 0 & 0 & -0.037 & 0.596 & -0.092 \\
\hline & Life expectancy & 0.557 & 0.02 & 1.095 & 0.2 & 0.042 & 0.476 \\
\hline & Prevalence & -0.008 & -0.013 & -0.004 & -0.319 & 0.001 & 0.185 \\
\hline
\end{tabular}

$R_{\text {unadjusted }}^{2}=0.153(F=23.225,(d f 130), p<0.001) . R_{\text {adjusted }}^{2}=0.443(F=19.071,(d f 125), p<0.001)$

$\mathrm{B}$, unstandardized coefficient; Beta, standardized coefficient; $\mathrm{Cl}$, confidence interval 
included ( $p=0.209)$. Other variables in the adjusted analysis showed a significant association with HAQ scores. Health expenditure was associated with a 0.008 point increase in HAQ score for each additional per capita dollar $(p<0.001)$, life expectancy was associated with a 1.371 point increase with each additional year of life $(p<0.001)$ and prevalence of hypertensive heart disease was associated with a 0.044 point decrease in HAQ score for each additional 100,000 people diagnosed with hypertensive heart disease $(p<0.001)$ (Table 4$)$.

\section{Discussion}

The number of medicines used to treat cerebrovascular disease and hypertensive heart disease included in national essential medicines lists was associated with amenable mortality, but the association was not present when country characteristics such as health spending were accounted for.

Our findings suggest that increases in a country's health expenditure may improve HAQ scores for cardiovascular disease. Fullman et al., (2018) found that health spending per capita was strongly correlated with HAQ Index performance, however there was a large variation in score within similar levels of spending [4]. Government spending as a fraction of total health spending was also positively correlated with $\mathrm{HAQ}$ Index performance [4]. Per-capita health expenditure is inadequate to pay for basic healthcare interventions in some low-income countries $[20,21]$. For the countries included in this study, 62 countries' (of the 131 total countries; one country had no data) per-capita government expenditure on health was less than the minimum required for basic effective public-health system [20]. A modest increase in public spending, efficient resource use and an investment in prevention programs is necessary for addressing inequity in healthcare [21]. It is also possible that higher healthcare spending would allow countries to purchase a better selection of medicines which may, in turn, lead to better health outcomes or higher spending could increase the availability of essential medicines.
We suspect that barriers within the healthcare system are particularly important for cardiovascular health. Inequity exists within the implementation of cost-effective interventions and the provision of care for cardiovascular disease predominantly in low-income countries where health systems may not be adequately equipped for providing chronic disease care [21]. For example, in Kenya, cardiovascular medicines can only be prescribed by physicians, [22] however it can be difficult for patients to access physicians due to a lack of effective referral networks [23] and a shortage of physicians making it difficult to contend with the disease burden [22]. Therefore, patients may be entering the healthcare system but not receiving proper cardiovascular care.

Other factors, such as quality of care, may impact mortality from cardiovascular disease. A study of 137 low- and middle-income countries found that amenable mortality outcomes were predominantly due to poor quality healthcare ( $84 \%$ of cardiovascular deaths amenable to healthcare), while the remaining $16 \%$ was due to non-utilization of healthcare [24]. This study shows that cardiovascular deaths for people entering the healthcare system are predominantly driven by poor quality of care. Therefore quality of care may account for some of the observed differences in amendable mortality and this would attenuate any real relationship between medicine selection and health outcomes.

\section{Strengths and limitations}

This was the first study we are aware of to compare NEML medications listings for cardiovascular diseases on a large scale. As a cross-sectional study, it would be inappropriate to draw causal conclusions about a relationship between medicine coverage scores and HAQ scores. Studying these associations over time may help solidify the conclusions drawn in this cross-sectional study. Applying a global medicine coverage score calculation represents a number of challenges. The score does not account for medicines that are therapeutically interchangeable within a class; theoretically, only one

Table 4 Hypertensive heart disease: medicine coverage score

\begin{tabular}{llllllrr}
\hline & Variable & B & $95 \%$ Cl lower bound & $95 \%$ Cl upper bound & Beta & P-value & Pearson correlation \\
\hline Unadjusted & Medicine coverage score & 0.621 & 0.312 & 0.929 & 0.331 & $<0.001$ & 0.331 \\
Adjusted & Medicine coverage score & 0.204 & -0.116 & 0.524 & 0.11 & 0.209 & 0.324 \\
& Health expenditure & 0.008 & 0.004 & 0.013 & 0.346 & $<0.001$ & 0.533 \\
& Population & $2.073 \mathrm{E}-06$ & 0 & 0 & 0.019 & 0.782 & -0.009 \\
& Life expectancy & 1.371 & 0.829 & 1.913 & 0.484 & $<0.001$ & 0.554 \\
& Prevalence & -0.044 & -0.063 & -0.026 & -0.402 & $<0.001$ & 0.084 \\
\hline
\end{tabular}

$R_{\text {unadjusted }}^{2}=0.109(F=15.846,(d f 130), p<0.001) . R_{\text {adjusted }}^{2}=0.454(F=19.963,(d f 125), p<0.001)$

$\mathrm{B}$, unstandardized coefficient; Beta, standardized coefficient; $\mathrm{Cl}$, confidence interval 
medicine in the class needs to be present for treatment, and the others are redundant. However, listing more than one medicine in a class can be beneficial in certain circumstances, for example in the case of drug recalls or shortages. In addition, there are no guidelines for the number of medicines needed in a class for proper coverage so we opted to include any that were listed in the country score. Although there are limitations to creating a medicine coverage score, our approach that was based on total medicines listed on NEMLs, allowed for an overall score that could be compared across many countries. The HAQ Index and GEM database both have their own limitations, which can be found in their respective articles $[4,10]$.

\section{Conclusions}

The number of medicines relevant to cardiovascular disease included in NEMLs is associated with amenable cardiovascular mortality but this association is not present when accounting for country attributes such as national healthcare spending. Country attributes may influence essential medicine listing which can impact health outcomes.

\section{Abbreviations}

ATC: Anatomical Therapeutic Chemical Classification; GEM: Global essential medicines; HAQ: Healthcare access and quality; NCD: Non-communicable disease; NEML: National essential medicines list; WHO: World Health Organization; WHO Model List: WHO model list of essential medicines.

\section{Supplementary Information}

The online version contains supplementary material available at https://doi. org/10.1186/s12872-021-01955-1.

\section{Additional file 1}

Acknowledgements

Not applicable.

\section{Authors' contributions}

LS, NP and SF contributed to the study conceptualization and design; LS and DM contributed to data collection; all authors contributed to data analysis and interpretation; LS drafted the manuscript. All authors read and approved the final manuscript.

\section{Funding}

NP reports grants from the Canadian Institutes of Health Research (CIHR), Ontario SPOR Support Unit, St Michael's Hospital Foundation, and Canada Research Chairs Program. The funders were not involved in the study design, data collection, analysis, interpretation of the research or writing of the manuscript. LS, SF and DM have no funding to declare.

\section{Availability of data and materials}

The datasets analyzed during the current study are publicly available in the GEM database (Persaud et al. [10]) and in Fullman et al. [4]. (https://doi.org/10. 6084/m9.figshare.7814246.v1; https://www.thelancet.com/journals/lancet/ article/PIIS0140-6736(18)30994-2/fulltext)

\section{Declarations}

Ethics approval and consent to participate

Not applicable.

\section{Consent for publication}

Not applicable.

\section{Competing interests}

NP reports grants from the Canadian Institutes of Health Research (CIHR), Ontario SPOR Support Unit, St Michael's Hospital Foundation, and Canada Research Chairs Program. LS, SF and DM have no competing interests to declare.

\section{Author details}

${ }^{1}$ MAP Centre for Urban Health Solutions, St. Michael's Hospital, Toronto, ON, Canada. ${ }^{2}$ Athabasca University, Athabasca, AB, Canada. ${ }^{3}$ Department of Family and Community Medicine, University of Toronto, Toronto, ON, Canada.

Received: 13 October 2020 Accepted: 14 March 2021

Published online: 25 March 2021

\section{References}

1. GBD Compare | IHME Viz Hub. 2018 [cited 2018 Sep 27]. https://vizhub. healthdata.org/gbd-compare/.

2. Bazargani UM, de Boer A, Leufkens HGM, Mantel-Teeuwisse AK. Selection of essential medicines for the prevention and treatment of cardiovascular diseases in low and middle income countries. BMC Cardiovasc Disord. 2018;25(18):126.

3. Abegunde D. Essential Medicines for Non-Communicable Diseases (NCDs) [Internet]. Geneva; 2011. Available from: https://www.who.int/ medicines/areas/policy/access_noncommunicable/en/.

4. Fullman N, Yearwood J, Abay SM, Abbafati C, Abd-Allah F, Abdela J, et al. Measuring performance on the Healthcare Access and Quality Index for 195 countries and territories and selected subnational locations: a systematic analysis from the Global Burden of Disease Study 2016. Lancet. 2018:391:2236-71.

5. The World Health Organization. Briefing document: essential medicines for non-communicable diseases (NCDs). 2011. https://www.who.int/ medicines/areas/policy/access_noncommunicable/NCDbriefingdocu ment.pdf?ua=.

6. The World Health Organization. WHO policy perspectives on medicines equitable access to essential medicines: a framework for collective action 8. 2004. http://apps.who.int/medicinedocs/pdf/s4962e/s4962e.pdf.

7. The World Health Organization. HEARTS Technical Package for Cardiovascular disease management in primary health care. 2016. https://apps. who.int/iris/bitstream/handle/10665/252661/9789241511377-eng.pdf; jsessionid=A6EF6593B92AE82AEB8C4AD28DE606EA? sequence $=1$.

8. The World Health Organization. Essential medicines and health products information portal. 2018. http://apps.who.int/medicinedocs/static/Publi cSubcollections/National-Essential-Medicines-Lists-NEMLs-Repository/.

9. Mahmić-Kaknjo M, Jeličić-Kadić A, Utrobičić A, Chan K, Bero L, Marušić A. Essential medicines availability is still suboptimal in many countries: a scoping review. J Clin Epidemiol. 2018;98:41-52. https://doi.org/10. 1016/j.jclinepi.2018.02.006.

10. Persaud N, Jiang M, Shaikh R, Bali A, Oronsaye E, Woods H, et al. Comparison of essential medicines lists in 137 countries. Bull World Health Organ. 2019;97:394.

11. Persaud N, Jiang M, Shaikh R, Bali A, Oronsaye E, Woods H, et al. Comparison of essential medicines lists in 137 countries-Supplementary figure and tables. figshare. 2019 [cited 2020 Aug 25]. https://doi.org/10.6084/ m9.figshare.7814246.v1.

12. The World Health Organization. Prevention and control of noncommunicable diseases: guidelines for primary health care in low resource settings. 2012;68.

13. The World Health Organization. Package of essential noncommunicable (PEN) disease interventions for primary health care in low-resource settings. 2010. http://www.who.int/cardiovascular_diseases. 
14. The World Health Organization. Tackling NCDS - Best Buys. 2017; https:// apps.who.int/iris/bitstream/handle/10665/259232/WHO-NMH-NVI-17.9eng.pdf;jsessionid=8BC070A284E32E92FA7273189F82E9DD?seque nce $=1$.

15. American Heart Association. Cardiac medications. 2019 [cited 2019 Oct 5]. https://www.heart.org/en/health-topics/heart-attack/treatment-of-aheart-attack/cardiac-medications.

16. The World Health Organization. WHO model list of essential medicines 20th edition. 2017. http://www.who.int/medicines/publications/essen tialmedicines/20th_EML2017.pdf.

17. The World Health Organization. WHO | Global Health Observatory (GHO) data. WHO. World Health Organization; 2018 [cited 2018 Dec 2]. http:// www.who.int/gho/en/.

18. WHO Collaborating Centre for Drug Statistics Methodology. ATC/DDD Index 2018. Norwegian Institute of Public Health; 2018.

19. The World Bank. GDP (current US\$) | Data. [cited 2018 Dec 2]. https://data. worldbank.org/indicator/NY.GDP.MKTP.CD.

20. Backman G, Hunt P, Khosla R, Jaramillo-Strouss C, Fikre BM, Rumble C, et al. Health systems and the right to health: an assessment of 194 countries. Lancet. 2008;372(9655):2047-85.
21. Mendis S, Banerjee A. Equity, social determinants and public health programmes. In: Blas E, Kurup S, editors. The World Health Organization; 2010. p. 31-48. https://apps.who.int/iris/bitstream/handle/10665/44289/ 9789241563970_eng.pdf?sequence=1\&isAllowed=y.

22. Vedanthan R, Tuikong N, Kofler C, Blank E, Kamano JH, Naanyu V, et al. Barriers and facilitators to nurse management of hypertension: a qualitative analysis from western Kenya. Ethn Dis. 2016;26(3):315-22.

23. Mercer T, Njuguna B, Bloomfield GS, Dick J, Finkelstein E, Kamano J, et al. Strengthening referral networks for management of hypertension across the health system (STRENGTHS) in western Kenya: a study protocol of a cluster randomized trial. Trials. 2019;20(1):554.

24. Kruk ME, Gage AD, Joseph NT, Danaei G, García-Saisó S, Salomon JA. Mortality due to low-quality health systems in the universal health coverage era: a systematic analysis of amenable deaths in 137 countries. Lancet. 2018;392(10160):2203-12.

\section{Publisher's note}

Springer Nature remains neutral with regard to jurisdictional claims in published maps and institutional affiliations.
Ready to submit your research? Choose BMC and benefit from:

- fast, convenient online submission

- thorough peer review by experienced researchers in your field

- rapid publication on acceptance

- support for research data, including large and complex data types

- gold Open Access which fosters wider collaboration and increased citations

- maximum visibility for your research: over 100M website views per year

At BMC, research is always in progress.

Learn more biomedcentral.com/submissions 